\title{
THE NONLINEAR ERGODIC THEOREM FOR ASYMPTOTICALLY NONEXPANSIVE MAPPINGS IN BANACH SPACES
}

\author{
KOK-KEONG TAN AND HONG-KUN XU
}

(Communicated by Palle E. T. Jorgensen)

\begin{abstract}
Let $X$ be a uniformly convex Banach space with a Frechet differentiable norm, $C$ a bounded closed convex subset of $X$, and $T: C \rightarrow C$ an asymptotically nonexpansive mapping. It is shown that for each $x$ in $C$, the sequence $\left\{T^{n} x\right\}$ is weakly almost-convergent to a fixed point $y$ of $T$, i.e., $(1 / n) \sum_{i=0}^{n-1} T^{k+i} x \rightarrow y$ weakly as $n$ tends to infinity uniformly in $k=0,1,2, \ldots$.
\end{abstract}

\section{INTRODUCTION}

Let $C$ be a nonempty subset of a Banach space $X$. A mapping $T: C \rightarrow C$ is said to be asymptotically nonexpansive [5] if there exists a sequence $\left\{L_{n}\right\}$ of positive real numbers with $\limsup _{n \rightarrow \infty} L_{n} \leq 1$ such that

$$
\left\|T^{n} x-T^{n} y\right\| \leq L_{n}\|x-y\|
$$

for all $x, y$ in $C$ and $n=1,2, \ldots$ If $C$ is assumed to be a bounded closed convex subset of a uniformly convex Banach space, then every asymptotically nonexpansive mapping $T: C \rightarrow C$ has a fixed point in $C$ (see [5]). An asymptotically nonexpansive mapping $T$ is said to be nonexpansive if $L_{n}=1$ for all $n \geq 1$. The first nonlinear ergodic theorem was proved by Baillon [1] in a Hilbert space: Let $C$ be a bounded closed convex subset of a Hilbert space $H$ and $T: C \rightarrow C$ a nonexpansive mapping; then for each $x$ in $C$, the Cesaro means

$$
S_{n}(x)=(1 / n) \sum_{i=0}^{n-1} T^{i} x, \quad n=1,2, \ldots
$$

converge weakly to a fixed point of $T$. In this case, letting $P(x)=w-$ $\lim S_{n}(x)$, we define a nonexpansive retraction from $C$ onto the fixed point set $F(T)$ of $T$ such that

$$
\text { (a) } \quad T P(x)=P T(x)=P(x) \text { and }\left(\text { b) } P x \in \bigcap_{n=0}^{\infty} \overline{\operatorname{co}}\left\{T^{i} x: i \geq n\right\}\right.
$$

Received by the editors July 16, 1990.

1980 Mathematics Subject Classification (1985 Revision). Primary 47H10, 47H09.

Key words and phrases. Asymptotically nonexpansive mapping, nonlinear ergodic theorem, fixed point. 
for all $x$ in $C$, where $\overline{c o}(A)$ denotes the closure of the convex hull of $A$. Baillon's theorem was generalized to the Banach space setting by Reich [10], Bruck [3], and Hirano [6]. On the other hand, Baillon's theorem was shown to be true by Hirano and Takahashi [7] for an asymptotically nonexpansive mapping and by You and $\mathrm{Xu}$ [12] for a wider class of mappings (i.e., mappings of asymptotically nonexpansive type [8]).

The objective of this paper is to prove the weak almost-convergence of the sequence $\left\{T^{n} x\right\}$ of an asymptotically nonexpansive mapping $T$ in a uniformly convex Banach space $X$ that has a Frechet differentiable norm, thus extending some previous results of Reich [10], Bruck [3], Hirano [6], Hirano and Takahashi [7], and You and $\mathrm{Xu}$ [12] to an asymptotically nonexpansive mapping or to the Banach space setting.

\section{LEMMAS}

Let $C$ be a bounded closed convex subset of a Banach space $X$ and $T: C \rightarrow$ $C$ an asymptotically nonexpansive mapping. In the sequel, we shall always assume without loss of any generality that $L_{n} \geq 1$ for all $n \geq 1$ and hence $\lim _{n \rightarrow \infty} L_{n}=1$.

Lemma 2.1. For every $x, y$ in $C$, the $\lim _{n \rightarrow \infty}\left\|T^{n} x-T^{n} y\right\|$ exists and $\| T^{m} x-$ $T^{m} y\left\|\geq \lim _{n \rightarrow \infty}\right\| T^{n} x-T^{n} y \|$ for all $m \geq 0$.

Proof. Since $T$ is asymptotically nonexpansive, we have

$$
\left\|T^{n+m} x-T^{n+m} y\right\| \leq L_{n}\left\|T^{m} x-T^{m} y\right\|
$$

for all $n, m \geq 0$. It then follows that

$$
\limsup _{n \rightarrow \infty}\left\|T^{n} x-T^{n} y\right\|=\underset{n \rightarrow \infty}{\limsup }\left\|T^{n+m} x-T^{n+m} y\right\| \leq\left\|T^{m} x-T^{m} y\right\|
$$

for each integer $m \geq 0$. This completes the proof.

Lemma 2.2 (Bruck [4]). Suppose $C$ is a bounded closed convex subset of a uniformly convex Banach space. Then there exists a strictly increasing continuous convex function $g:[0, \infty) \rightarrow[0, \infty)$ with $g(0)=0$ such that

$$
g\left(\left\|T\left(\sum_{i=1}^{n} \lambda_{i} x_{i}\right)-\sum_{i=1}^{n} \lambda_{i} T x_{i}\right\|\right) \leq \max _{1 \leq i, j \leq n}\left(\left\|x_{i}-x_{j}\right\|-\left\|T x_{i}-T x_{j}\right\|\right)
$$

for any nonexpansive $T: C \rightarrow X$, any $x_{1}, \ldots, x_{n}$ in $C$ and any $\lambda_{1}, \ldots, \lambda_{n} \geq$ 0 such that $\lambda_{1}+\cdots+\lambda_{n}=1$.

Lemma 2.3. Let $C$ be a bounded closed convex subset of a uniformly convex Banach space $X$. Suppose $\left\{x_{k}\right\}$ is in $C$ such that $x_{k} \rightarrow x$ weakly and $\varlimsup_{n}\left(\overline{\lim }_{k}\left\|x_{k}-T^{n} x_{k}\right\|\right)=0$. Then $x=T x$.

Proof. For an arbitrary $\varepsilon>0$, choose $n_{0}$ such that

$$
\varlimsup_{k}\left\|x_{k}-T^{n} x_{k}\right\|<\varepsilon
$$

for all $n \geq n_{0}$. Since $x_{k} \rightarrow x$ weakly, $x \in \overline{\mathrm{co}}\left\{x_{i}: i \geq k\right\}$ for each $k$ and hence one can choose for each $k$, a $y_{k}=\sum_{i=1}^{m} \lambda_{i} x_{i+k}$ with $\lambda_{i} \geq 0, \sum_{i=1}^{m} \lambda_{i}=1$ such that

$$
\left\|x-y_{k}\right\|<1 / k
$$


Now for an arbitrary but fixed $n \geq n_{0}$, by (2.1) we can find a $k_{0}$ such that

$$
\left\|x_{k}-T^{n} x_{k}\right\|<\varepsilon \text { for all } k \geq k_{0} \text {. }
$$

It then follows from (2.3) and Lemma 2.2 that for $k \geq k_{0}$,

$$
\begin{aligned}
\left\|T^{n} y_{k}-y_{k}\right\| & \leq\left\|T^{n}\left(\sum_{i=1}^{m} \lambda_{i} x_{i+k}\right)-\sum_{i=1}^{m} \lambda_{i} T^{n} x_{i+k}\right\|+\sum_{i=1}^{m} \lambda_{i}\left\|T^{n} x_{i+k}-x_{i+k}\right\| \\
& \leq L_{n} g^{-1}\left(\max _{1 \leq i, j \leq m}\left(\left\|x_{i+k}-x_{j+k}\right\|-L_{n}^{-1}\left\|T^{n} x_{i+k}-T^{n} x_{j+k}\right\|\right)\right)+\varepsilon \\
& \leq L_{n} g^{-1}\left(\max _{1 \leq i, j \leq m}\left(\left\|x_{i+k}-x_{j+k}\right\|-\left\|T^{n} x_{i+k}-T^{n} x_{j+k}\right\|\right)\right. \\
& \left.+\left(1-L_{n}^{-1}\right) d\right)+\varepsilon, \quad \text { where } d=\operatorname{diam}(C) .
\end{aligned}
$$

Since

$$
\begin{aligned}
\left\|x_{i+k}-x_{j+k}\right\| & \leq\left\|x_{i+k}-T^{n} x_{i+k}\right\|+\left\|T^{n} x_{i+k}-T^{n} x_{j+k}\right\|+\left\|x_{j+k}-T^{n} x_{j+k}\right\| \\
& \leq\left\|T^{n} x_{i+k}-T^{n} x_{j+k}\right\|+2 \varepsilon
\end{aligned}
$$

by $(2.3)$, we obtain

$$
\left\|T^{n} y_{k}-y_{k}\right\| \leq L_{n} g^{-1}\left(\left(1-L_{n}^{-1}\right) d+2 \varepsilon\right)+\varepsilon
$$

for all $k \geq k_{0}$ and hence

$$
\varlimsup_{k}\left\|T^{n} y_{k}-y_{k}\right\| \leq L_{n} g^{-1}\left(2 \varepsilon+\left(1-L_{n}^{-1}\right) d\right)+\varepsilon
$$

for all $n \geq n_{0}$. Therefore,

$$
\begin{aligned}
\left\|T^{n} x-x\right\| & \leq \varlimsup_{k}\left(\left\|T^{n} x-T^{n} y_{k}\right\|+\left\|T^{n} y_{k}-y_{k}\right\|+\left\|y_{k}-x\right\|\right) \\
& \leq \varlimsup_{k}\left(\left(1+L_{n}\right)\left\|y_{k}-x\right\|+\left\|T^{n} y_{k}-y_{k}\right\|\right) \\
& \leq L_{n} g^{-1}\left(2 \varepsilon+\left(1-L_{n}^{-1}\right) d\right)+\varepsilon \rightarrow 0 \text { as } n \rightarrow \infty \text { and } \varepsilon \rightarrow 0 .
\end{aligned}
$$

This shows $T^{n} x \rightarrow x$ strongly and thus $T x=x$ by continuity of $T$. The proof is complete.

The following is a partial extension of a result of Browder [2] to asymptotically nonexpansive mappings.

Corollary 2.1. Let $C$ be a bounded closed convex subset of a uniformly convex Banach space and $T: C \rightarrow C$ an asymptotically nonexpansive mapping. Then $(I-T)$ is demiclosed at zero, i.e., for any sequence $\left\{x_{n}\right\}$ in $C$, the conditions $x_{n} \rightarrow x$ weakly and $(I-T) x_{n} \rightarrow 0$ strongly imply $(I-T) x=x$. (Here I denotes the identity operator on $X$.)

Notation. $F(T)$ is the set of fixed points of $T$, i.e., $F(T)=\{x \in C: T x=x\}$. $\rightarrow$ stands for strong convergence and $\rightarrow$ for weak convergence.

\section{THE NONLINEAR ERGODIC THEOREM}

In this section we prove the nonlinear ergodic theorem for asymptotically nonexpansive mappings in Banach spaces. The main result, Theorem 3.1 generalizes results of Reich [10], Bruck [3], Hirano [6], Hirano and Takahashi [7], and You and $\mathrm{Xu}$ [12] to asymptotically nonexpansive mappings or to the Banach space setting. 
Proposition 3.1. Let $C$ be a bounded closed convex subset of a uniformly convex Banach space $X$ and $T: C \rightarrow C$ an asymptotically nonexpansive mapping. Then for each $x$ in $C$, each integer $n \geq 1$ and an arbitrary $\varepsilon>0$, there exist an $i_{n}$ and $a k_{0}$ depending only on $n$ and $\varepsilon$ respectively such that

$$
\left\|T^{k} S_{n} T^{i} x-S_{n} T^{k} T^{i} x\right\| \leq(1+\varepsilon) g^{-1}(1 / n+\varepsilon M)
$$

for all $k \geq k_{0}, i \geq i_{n}$, and $n \geq 1$, where

$$
S_{n}=(1 / n)\left(I+T+\cdots+T^{n-1}\right),
$$

$g$ is the function given in Lemma 2.2, and $M=\sup \left\{\left\|T^{n} x-T^{m} x\right\|: n, m \geq 0\right\}$.

Proof. By Lemma 2.2, we have

$$
\begin{aligned}
& g\left(L_{k}^{-1}\left\|T^{k} S_{n} T^{i} x-S_{n} T^{k} T^{i} x\right\|\right) \\
& \quad \leq \max _{0 \leq j, m \leq n-1}\left(\left\|T^{i+j} x-T^{i+m} x\right\|-L_{k}^{-1}\left\|T^{k+i+j} x-T^{k+i+m} x\right\|\right) \\
& \quad \leq \max _{0 \leq j, m \leq n-1}\left(\left\|T^{i+j} x-T^{i+m} x\right\|-\left\|T^{k+i+j} x-T^{k+i+m} x\right\|\right)+\left(1-L_{k}^{-1}\right) M .
\end{aligned}
$$

Now for a fixed integer $n \geq 1$ and an arbitrary $\varepsilon>0$, one can choose an $i_{n}$ and a $k_{0}$ depending only on $n$ and $\varepsilon$ respectively such that

$$
\left\|T^{i+j} x-T^{i+m} x\right\|<r_{j m}+1 / n
$$

for all $i \geq i_{n}$ and $j, m=0,1, \ldots, n-1$, where $r_{j m}=\lim _{i \rightarrow \infty}\left\|T^{i+j} x-T^{i+m} x\right\|$, and

$$
L_{k}<1+\varepsilon
$$

for all $k \geq k_{0}$. It then follows from (3.1), (3.2), and Lemma 2.1 that for $k \geq k_{0}$ and $i \geq i_{n}$

$$
\begin{aligned}
& \left\|T^{k} S_{n} T^{i} x-S_{n} T^{k} T^{i} x\right\| \\
& \quad \leq L_{k} g^{-1}\left(\max _{0 \leq j, m \leq n-1}\left(\left\|T^{i+j} x-T^{i+m} x\right\|-r_{j m}\right)+(\varepsilon /(1+\varepsilon)) M\right) \\
& \quad \leq(1+\varepsilon) g^{-1}(1 / n+\varepsilon M) .
\end{aligned}
$$

This completes the proof.

We also need the following special case of [Theorem 3.1, 11].

Proposition 3.2. Let $X$ be a uniformly convex Banach space having a Frechet differentiable norm, $C$ a bounded closed convex subset of $X$, and $T: C \rightarrow C$ an asymptotically nonexpansive mapping. Then for each $x$ in $C$, the set

$$
\bigcap_{n=0}^{\infty} \overline{\operatorname{co}}\left\{T^{i} x: i \geq n\right\} \cap F(T)
$$

consists of at most one point.

Following Lorentz [9], we say that a sequence $\left\{x_{n}\right\}$ is weakly almostconvergent to $x$ if

$$
\frac{1}{n} \sum_{i=0}^{n-1} x_{i+k}-x \quad \text { uniformly in } k=0,1, \ldots
$$


Theorem 3.1. Let $X$ be a uniformly convex Banach space having a Frechet differentiable norm, $C$ a bounded closed convex subset of $X$, and $T: C \rightarrow C$ an asymptotically nonexpansive mapping. Then for each $x$ in $C,\left\{T^{n} x\right\}$ is weakly almost-convergent to the unique point of the set $\bigcap_{n=0}^{\infty} \overline{\operatorname{co}}\left\{T^{i} x: i \geq n\right\} \cap F(T)$.

Proof. First note that $F(T)$ is nonempty by Goebel and Kirk [5]. By Proposition 3.1, we have

$$
\left\|T^{k} S_{n} T^{i_{n}} x-S_{n} T^{k} T^{i_{n}} x\right\| \leq(1+\varepsilon) g^{-1}(1 / n+\varepsilon M)
$$

for all $k \geq k_{0}$ and $n \geq 1$. Noticing

$$
\left\|S_{n} T^{k} T^{i_{n}} x-S_{n} T^{i_{n}} x\right\| \leq k M / n
$$

we obtain from (3.3) that

$$
\left\|T^{k} S_{n} T^{i_{n}} x-S_{n} T^{i_{n}} x\right\| \leq(1+\varepsilon) g^{-1}(1 / n+\varepsilon M)+k M / n
$$

for all $n \geq 1$ and $k \geq k_{0}$. Therefore, we get

$$
\varlimsup_{k}\left(\varlimsup_{n}\left\|T^{k} S_{n} T^{i_{n}} x-S_{n} T^{i_{n}} x\right\|\right)=0 \text {. }
$$

This, together with Lemma 2.3 and Proposition 3.2, implies

$$
S_{n} T^{i_{n}} x-y=\bigcap_{n=0}^{\infty} \overline{\operatorname{co}}\left\{T^{i} x: i \geq n\right\} \cap F(T) .
$$

By the same way as above, one can easily show that

$$
\left\|T^{k} S_{n} T^{i_{n}+p_{n}+i} x-S_{n} T^{i_{n}+p_{n}+i} x\right\| \leq(1+\varepsilon) g^{-1}(1 / n+\varepsilon M)+k M / n
$$

for all integers $n, p_{n}, i \geq 1$ and $k \geq k_{0}$. Together again with Lemma 2.3 and Proposition 3.2, we have for each $i \geq 0$,

$$
S_{n} T^{i_{n}+n p+i} x-y \text { uniformly in } p=0,1,2, \ldots \text {. }
$$

Now for $n$ and $m \geq i_{n}$, since

$$
\begin{aligned}
S_{m} T^{i} x & =\frac{1}{m} \sum_{k=0}^{m-1} T^{k} T^{i} x \\
& =\frac{1}{m}\left(\sum_{k=i_{n}+j n}^{m-1} T^{k+i} x+n\left(\sum_{k=0}^{j} S_{n} T^{i_{n}+n k+i} x\right)+\sum_{k=0}^{i_{n}} T^{k+i} x\right),
\end{aligned}
$$

where $m=j n+i_{n}+r, r<n$, we deduce from (3.5) that $S_{m} T^{i} x \rightarrow y$ uniformly in $i=0,1,2, \ldots$. This completes the proof.

Remark. Let us denote by $P x$ the limit of $\left\{S_{n}(x)\right\}$ in Theorem 3.1. Then it can be easily seen that $P$ is the unique nonexpansive retraction from $C$ onto $F(T)$ satisfying the properties (a) and (b) in the introduction.

Recall that $T$ is said to be weakly asymptotically regular at $x \in C$ if $T^{n} x-$ $T^{n+1} x-0$. The following theorem was proved by Reich [10] and Bruck [3] for nonexpansive mappings.

Theorem 3.2. Let $X, C$, and $T$ be as given in Theorem 3.1. Then $\left\{T^{n} x\right\}$ converges weakly to a fixed point of $T$ if and only if $T$ is weakly asymptotically regular at $x$.

Proof. Necessity is trivial and sufficiency follows from Theorem 3.1 and the fact that $x_{n}-x_{n+1}-0$ is a Tauberian condition for weak almost-convergence of $\left\{x_{n}\right\}$ (cf. Lorentz [9]). 


\section{REFERENCES}

1. J. B. Baillon, Un théorème de type ergodique pour les contractions non linéaires dans un espace de Hilbert, C. R. Acad. Sci. Paris Sér. I Math. 280 (1975), 1511-1514.

2. F. E. Browder, Semicontractive and semiaccretive nonlinear mappings in Banach spaces, Bull. Amer. Math. Soc. 74 (1968), 660-665.

3. R. E. Bruck, $A$ simple proof of the mean ergodic theorem for nonlinear contractions in Banach spaces, Israel J. Math. 32 (1979), 107-116.

4. _ On the convex approximation property and the asymptotic behavior of nonlinear contractions in Banach spaces, Israel J. Math. 34 (1981), 304-314.

5. K. Goebel and W. A. Kirk, A fixed point theorem for asymptotically nonexpansive mappings, Proc. Amer. Math. Soc. 35 (1972), 171-174.

6. N. Hirano, $A$ proof of the mean ergodic theorem for nonexpansive mappings in Banach spaces, Proc. Amer. Math. Soc. 78 (1980), 361-365.

7. N. Hirano and W. Takahashi, Nonlinear ergodic theorems for nonexpansive mappings in Hilbert spaces, Kodai Math. J. 2 (1979), 11-25.

8. W. A. Kirk, Fixed point theorems for non-Lipschitzian mappings of asymptotically nonexpansive type, Israel J. Math. 17 (1974), 339-346.

9. G. G. Lorentz, A contribution to the theory of divergent series, Acta Math. 80 (1948), 167190.

10. S. Reich, Weak convergence theorems for nonexpansive mappings in Banach spaces, J. Math. Anal. Appl. 67 (1979), 274-276.

11. K.-K. Tan and H.-K. Xu, Asymptotic behavior of nonlinear Lipschitzian semigroups in Banach spaces, submitted.

12. Z. Y. You and H.-K. Xu, The ergodic convergence theorem for mappings of asymptotically nonexpansive type, Chinese Ann. Math. Ser. A 11 (1990), 121-125.

Department of Mathematics, Statistics and Computing Science, Dalhousie University, Halifax, Nova Scotia, Canada B3H 3J5

Department of Mathematics, East China University of Chemical Technology, ShangHAI 200237, CHINA 